\title{
Decidable Synthesis of Programs with Uninterpreted Functions
}

\author{
Paul Krogmeier ${ }^{\left({ }^{\otimes}\right)}$ (D), Umang Mathur(D), Adithya Murali(D), P. Madhusudan, \\ and Mahesh Viswanathan
}

University of Illinois at Urbana-Champaign, Champaign, USA

\{paulmk2, umathur3, adithya5, madhu, vmahesh\}@illinois.edu

\begin{abstract}
We identify a decidable synthesis problem for a class of programs of unbounded size with conditionals and iteration that work over infinite data domains. The programs in our class use uninterpreted functions and relations, and abide by a restriction called coherence that was recently identified to yield decidable verification. We formulate a powerful grammar-restricted (syntax-guided) synthesis problem for coherent uninterpreted programs, and we show the problem to be decidable, identify its precise complexity, and also study several variants of the problem.
\end{abstract}

\section{Introduction}

Program synthesis is a thriving area of research that addresses the problem of automatically constructing a program that meets a user-given specification $[1,21,22]$. Synthesis specifications can be expressed in various ways: as input-output examples $[19,20]$, temporal logic specifications for reactive programs [44], logical specifications [1,4], etc. Many targets for program synthesis exist, ranging from transition systems [31,44], logical expressions [1], imperative programs [51], distributed transition systems/programs [38, 43, 45], filling holes in programs [51], or repairs of programs [49].

A classical stream of program synthesis research is one that emerged from a problem proposed by Church [13] in 1960 for Boolean circuits. Seminal results by Büchi and Landweber [9] and Rabin [48] led to a mature understanding of the problem, including connections to infinite games played on finite graphs and automata over infinite trees (see $[18,32]$ ). Tractable synthesis for temporal logics like LTL, CTL, and their fragments was investigated and several applications for synthesizing hardware circuits emerged $[6,7]$.

In recent years, the field has taken a different turn, tackling synthesis of programs that work over infinite domains such as strings [19,20], integers [1,51], and heaps [47]. Typical solutions derived in this line of research involve (a) bounding the class of programs to a finite set (perhaps iteratively increasing the class) and (b) searching the space of programs using techniques like symmetryreduced enumeration, SAT solvers, or even random walks [1,4], typically guided

Paul Krogmeier and Mahesh Viswanathan are partially supported by NSF CCF 1901069. Umang Mathur is partially supported by a Google PhD Fellowship.

(C) The Author(s) 2020

S. K. Lahiri and C. Wang (Eds.): CAV 2020, LNCS 12225, pp. 634-657, 2020.

https://doi.org/10.1007/978-3-030-53291-8_32 
by counterexamples (CEGIS) $[28,34,51]$. Note that iteratively searching larger classes of programs allows synthesis engines to find a program if one exists, but it does not allow one to conclude that there is no program that satisfies the specification. Consequently, in this stream of research, decidability results are uncommon (see Sect. 7 for some exceptions in certain heavily restricted cases).

In this paper we present, to the best of our knowledge, the first decidability results for program synthesis over a natural class of programs with iteration/recursion, having arbitrary sizes, and which work on infinite data domains. In particular, we show decidable synthesis of a subclass of programs that use uninterpreted functions and relations.

Our primary contribution is a decidability result for realizability and synthesis of a restricted class of imperative uninterpreted programs. Uninterpreted programs work over infinite data models that give arbitrary meanings to their functions and relations. Such programs satisfy their assertions if they hold along all executions for every model that interprets the functions and relations. The theory of uninterpreted functions and relations is well studied - classically, in 1929, by Gödel, where completeness results were shown [5] and, more recently, its decidable quantifier-free fragment has been exploited in SMT solvers in combination with other theories [8]. In recent work [39], a subclass of uninterpreted programs, called coherent programs, was identified and shown to have a decidable verification problem. Note that in this verification problem there are no user-given loop invariants; the verification algorithm finds inductive invariants and proves them automatically in order to prove program correctness.

In this paper, we consider the synthesis problem for coherent uninterpreted programs. The user gives a grammar $\mathcal{G}$ that generates well-formed programs in our programming language. The grammar can force programs to have assert statements at various points which collectively act as the specification. The program synthesis problem is then to construct a coherent program, if one exists, conforming to the grammar $\mathcal{G}$ that satisfies all assertions in all executions when running on any data model that gives meaning to function and relation symbols.

Our primary result is that the realizability problem (checking the existence of a program conforming to the grammar and satisfying its assertions) is decidable for coherent uninterpreted programs. We prove that the problem is 2EXPTIMEcomplete. Further, whenever a correct coherent program that conforms to the grammar exists, we can synthesize one. We also show that the realizability/synthesis problem is undecidable if the coherence restriction is dropped. In fact we show a stronger result that the problem is undecidable even for synthesis of straight-line programs (without conditionals and iteration)!

Coherence of programs is a technical restriction that was introduced in [39]. It consists of two properties, both of which were individually proven to be essential for ensuring that program verification is decidable. Intuitively, the restriction demands that functions are computed on any tuple of terms only once and that assumptions of equality come early in the executions. In more recent work [41], the authors extend this decidability result to handle map updates, and applied it to memory safety verification for a class of heap-manipulating programs on 
forest data-structures, demonstrating that the restriction of coherence is met in practice by certain natural and useful classes of programs.

Note that automatic synthesis of correct programs over infinite domains demands that we, at the very least, can automatically verify the synthesized program to be correct. The class of coherent uninterpreted programs identified in the work of [39] is the only natural class of programs we are aware of that has recursion and conditionals, works over infinite domains, and admits decidable verification. Consequently, this class is a natural target for proving a decidable synthesis result.

The problem of synthesizing a program from a grammar with assertions is a powerful formulation of program synthesis. In particular, the grammar can be used to restrict the space of programs in various ways. For example, we can restrict the space syntactically by disallowing while loops. Or, for a fixed $n$, by using a set of Boolean variables linear in $n$ and requiring a loop body to strictly increment a counter encoded using these variables, we can demand that loops terminate in a linear/polynomial/exponential number of iterations. We can also implement loops that do not always terminate, but terminate only when the data model satisfies a particular property, e.g., programs that terminate only on finite list segments, by using a skeleton of the form: while $(\mathrm{x} \neq \mathrm{y})\{\ldots ; \mathrm{x}:=\operatorname{next}(\mathrm{x})\}$. Grammar-restricted program synthesis can express the synthesis of programs with holes, used in systems like SKETCH [50], where the problem is to fill holes using programs/expressions conforming to a particular grammar so that the assertions in the program hold. Synthesizing programs or expressions using restricted grammars is also the cornerstone of the intensively studied SYGUS (syntax-guided synthesis) format $[1,52]^{1}$.

The proof of our decidability result relies on tree automata, a callback to classical theoretical approaches to synthesis. The key idea is to represent programs as trees and build automata that accept trees corresponding to correct programs. The central construction is to build a two-way alternating tree automaton that accepts all program trees of coherent programs that satisfy their assertions. Given a grammar $\mathcal{G}$ of programs (which has to satisfy certain natural conditions), we show that there is a regular set of program trees for the language of allowed programs $L(\mathcal{G})$. Intersecting the automata for these two regular tree languages and checking for emptiness establishes the upper bound. Our constructions crucially use the automaton for verifying coherent uninterpreted programs in [39] and adapt ideas from [35] for building two-way automata over program trees. Our final decision procedure is doubly-exponential in the number of program variables and linear in the size of the grammar. We also prove a matching lower bound by reduction from the acceptance problem for alternating exponential-space Turing machines. The reduction is non-trivial in that programs (which correspond to runs in the Turing machine) must simulate sequences of configurations, each of which is of exponential size, by using only polynomially-many variables.

\footnotetext{
${ }^{1}$ Note, however, that both SкEтch and SyGuS problems are defined using functions and relations that are interpreted using standard theories like arithmetic, etc., and hence of course do not have decidable synthesis.
} 
Recursive Programs, Transition Systems, and Boolean Programs: We study three related synthesis problems. First, we show that our results extend to synthesis of call-by-value recursive uninterpreted programs (with a fixed number of functions and fixed number of local/global variables). This problem is also 2EXPTIME-complete but is more complex, as even single executions simulated on the program tree must be split into separate copies, with one copy executing the summary of a function call and the other proceeding under the assumption that the call has returned in a summarized state.

We next examine a synthesis problem for transition systems. Transition systems are similar to programs in that they execute similar kinds of atomic statements. We allow the user to restrict the set of allowable executions (using regular sets). Despite the fact that this problem seems very similar to program synthesis, we show that it is an easier problem, and coherent transition system realizability and synthesis can be solved in time exponential in the number of program variables and polynomial in the size of the automata that restrict executions. We prove a corresponding lower bound to establish EXPTIME-completeness of this problem.

Finally, we note that our results also show, as a corollary, that the grammarrestricted realizability/synthesis problem for Boolean programs (resp. executionrestricted synthesis problem for Boolean transition systems) is decidable and is 2EXPTIME-complete (resp. EXPTIME-complete). These results for Boolean programs are themselves new. The lower bound results for these problems hence show that coherent program/transition-system synthesis is not particularly harder than Boolean program synthesis for uninterpreted programs. Grammarrestricted Boolean program synthesis is an important problem which is addressed by many practical synthesis systems like Sketch [50].

Due to space restrictions, we present only proof gists for main results in the paper. All the complete proofs can be found in our technical report [30].

\section{Examples}

We will begin by looking at several examples to gain some intuition for uninterpreted programs.

Example 1. Consider the program in Fig. 1 (left). This program has a hole ' $\langle\langle$ ?? $|$ Cannot $\ldots\rangle\rangle$ ' that we intend to fill with a sub-program so that the entire program (together with the contents of the hole) satisfies the assertion at the end. The sub-program corresponding to the hole is allowed to use the variable cipher as well as some additional variables $\mathrm{y}_{1}, \ldots, \mathrm{y}_{n}$ (for some fixed $n$ ), but is not allowed to refer to key or secret in any way. Here we also restrict the hole to exclude while loops. This example models the encryption of a secret message secret with a key key. The assumption in the second line of the program models 


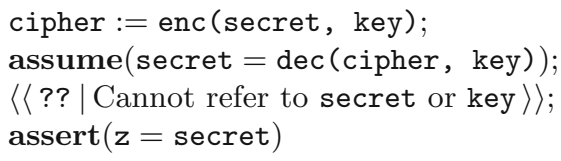

Decrypting a ciphertext

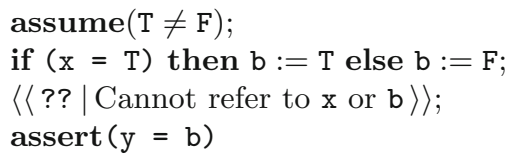

Synthesis with incomplete information

Fig. 1. Examples of programs with holes

the fact that the secret message can be decrypted from cipher and key. Here, the functions enc and dec are uninterpreted functions, and thus the program we are looking for is an uninterpreted program. For such a program, the assertion "assert(z = secret)" holds at the end if it holds for all models, i.e, for all interpretations of enc and dec and for all initial values of the program variables secret, key, cipher, and $\mathrm{y}_{1}, \ldots, \mathrm{y}_{n}$. With this setup, we are essentially asking whether a program that does not have access to key can recover secret. It is not hard to see that there is no program which satisfies the above requirement. The above modeling of keys, encryption, nonces, etc. is common in algebraic approaches to modeling cryptographic protocols $[15,16]$.

Example 2. The program in Fig. 1 (right) is another simple example of an unrealizable specification. The program variables here are $\mathrm{x}, \mathrm{b}$, and $\mathrm{y}$. The hole in this partial program is restricted so that it cannot refer to $\mathrm{x}$ or $\mathrm{b}$. It is easy to phrase the question for synthesis of the complete program in terms of a grammar. The restriction on the hole ensures that the synthesized code fragment can neither directly check if $\mathrm{x}=\mathrm{T}$, nor indirectly check via $\mathrm{b}$. Consequently, it is easy to see that there is no program for the hole that can ensure $\mathrm{y}$ is equal to $\mathrm{b}$. We remark that the code at the hole, apart from not being allowed to examine some variables, is also implicitly prohibited from looking at the control path taken to reach the hole. If we could synthesize two different programs depending on the control path taken to reach the hole, then we could set $\mathrm{y}:=\mathrm{T}$ when the then-branch is taken and set $\mathrm{y}:=\mathrm{F}$ when the else-branch is taken. Program synthesis requires a control-flow independent decision to be made about how to fill the hole. In this sense, we can think of the hole as having only incomplete information about the executions for which it must be correct. This can be used to encode specifications using complex ghost code, as we show in the next examples. In Sect.6, we explore a slightly different synthesis problem, called transition system synthesis, where holes can be differently instantiated based on the history of an execution.

Example 3. In this example, we model the synthesis of a program that checks whether a linked list pointed to by some node $\mathrm{x}$ has a key $\mathrm{k}$. We model a next pointer with a unary function next and we model locations using elements in the underlying data domain.

Our formalism allows only for assert statements to specify desired program properties. In order to state the correctness specification for our desired 
list-search program, we interleave ghost code into the program skeleton; we distinguish ghost code fragments by enclosing them in 'dashed boxes $;$. The skeleton in Fig. 2 has a loop that advances the pointer variable $\mathrm{x}$ along the list until NIL is reached. We model NIL with an immutable program variable. The first hole ' $\left\langle\left\langle\right.\right.$ ?? $\left.\left.{ }^{(1)}\right\rangle\right\rangle$ ' before the while-loop and the second hole ' $\left\langle\left\langle\right.\right.$ ?? $\left.{ }^{(2)}\right\rangle$ ' within the while-loop need to be filled so that the assertion at the end is satisfied. We use three ghost variables in the skeleton: $\mathrm{g}_{\text {ans }}, \mathrm{g}_{\mathrm{witness}}$, and $\mathrm{g}_{\text {found }}$. The ghost variable $\mathrm{g}_{\text {ans }}$ evaluates to whether we expect to find $\mathrm{k}$ in the list or not, and hence at the end the skeleton asserts that the Boolean variable $\mathrm{b}$ computed by the holes is precisely gans. The holes are restricted to not look at the ghost variables.

Now, notice that the skeleton needs to check that the answer $g_{\text {ans }}$ is indeed correct. If $g_{\text {ans }}$ is not $T$, then we add the assumption that $\operatorname{key}(\mathrm{x}) \neq \mathrm{k}$ in each iteration of the loop, hence ensuring the key is not present. For ensuring correctness in the case gans $=\mathrm{T}$, we need two more ghost variables $g_{w i t n e s s}$ and $g_{\text {found }}$. The variable $g_{w i t n e s s}$ witnesses the precise location in the list that holds the key $\mathrm{k}$, and variable $\mathrm{g}_{\text {found }}$ indicates whether the location at $\mathrm{g}_{\text {witness }}$ belongs to the list pointed to by $\mathrm{x}$. Observe that this specification can be realized by filling ' $\left\langle\left\langle\right.\right.$ ?? $\left.\left.{ }^{(1)}\right\rangle\right\rangle$ ' with " $\mathrm{b}:=\mathrm{F}$ " and ' $\left\langle\left\langle\right.\right.$ ?? $\left.\left.{ }^{(2)}\right\rangle\right\rangle$ ' with 'if key $(\mathrm{x})=\mathrm{k}$ then $\mathrm{b}:=$ T", for instance. Furthermore, this program is coherent [39] and hence our decision procedure will answer in the affirmative and synthesize code for the holes.

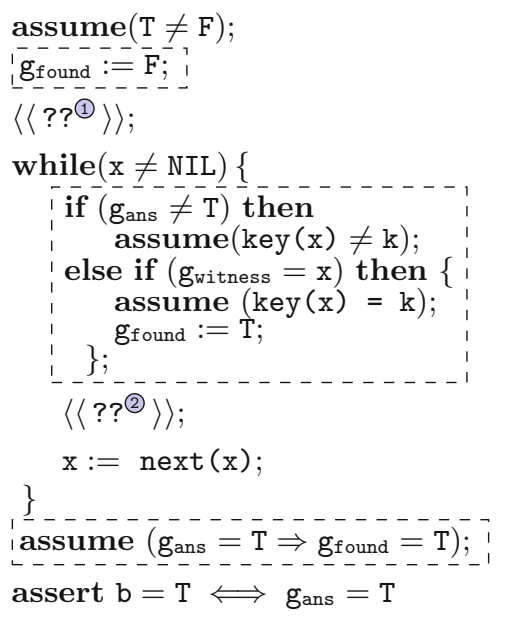

Fig. 2. Skeleton with ghost code

In fact, our procedure will synthesize a representation for all possible ways to fill the holes (thus including the solution above) and it is therefore possible to enumerate and pick specific solutions. It is straightforward to formulate a grammar which matches this setup. As noted, we must stipulate that the holes do not use the ghost variables.

Example 4. Consider the same program skeleton as in Example 3, but let us add an assertion at the end: "assert ( $\left.\mathrm{b}=\mathrm{T} \Rightarrow \mathrm{z}=\mathrm{g}_{\text {witness }}\right)$ ", where $\mathrm{z}$ is another program variable. We are now demanding that the synthesized code also find a location $\mathrm{z}$, whose key is $\mathrm{k}$, that is equal to the ghost location $\mathrm{g}_{\mathrm{witness}}$, which is guessed nondeterministically at the beginning of the program. This specification is unrealizable: for a list with multiple locations having the key $\mathrm{k}$, no matter what the program picks we can always take gwitness to be the other location with key $\mathrm{k}$ in the list, thus violating the assertion. Our decision procedure will report in the negative for this specification.

Example 5 (Input/Output Examples). We can encode input/output examples by adding a sequence of assignments and assumptions that define certain models at 
the beginning of the program grammar. For instance, the sequence of statements in Fig. 3 defines a linked list of two elements with different keys.

We can similarly use special variables to define the output that we expect in the case of each model. And as we saw in the ghost code of Fig. 2, we can use fresh variables to introduce nondeterministic choices, which the grammar can use to pick an example model nondeterministically. Thus when the synthesized program is executed on the chosen model it computes the expected answer. This has the effect of requiring a solution that generalizes across models. See [30] for a more detailed example.

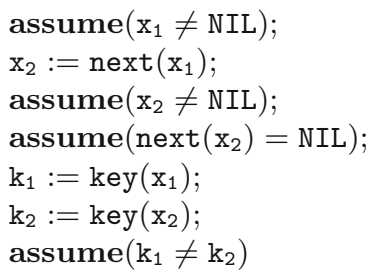

Fig. 3. An example model

\section{Preliminaries}

In this section we define the syntax and semantics of uninterpreted programs and the (grammar-restricted) uninterpreted program synthesis problem.

Syntax. We fix a first order signature $\Sigma=(\mathcal{F}, \mathcal{R})$, where $\mathcal{F}$ and $\mathcal{R}$ are sets of function and relation symbols, respectively. Let $V$ be a finite set of program variables. The set of programs over $V$ is inductively defined using the following grammar, with $f \in \mathcal{F}, R \in \mathcal{R}$ (with $f$ and $R$ of the appropriate arities), and $x, y, z_{1}, \ldots, z_{r} \in V$.

$$
\begin{aligned}
\langle\text { stmt }\rangle_{V}::= & \text { skip }|x:=y| x:=f\left(z_{1}, \ldots, z_{r}\right) \mid \\
& \text { assume }\left(\langle\text { cond }\rangle_{V}\right) \mid \text { assert }\left(\langle\text { cond }\rangle_{V}\right) \mid\langle\text { stmt }\rangle_{V} ;\langle\text { stmt }\rangle_{V} \mid \\
& \text { if }\left(\langle\text { cond }\rangle_{V}\right) \text { then }\langle\text { stmt }\rangle_{V} \text { else }\langle\text { stm }\rangle_{V} \mid \text { while }\left(\langle\text { cond }\rangle_{V}\right)\langle\text { stmt }\rangle_{V} \\
\langle\text { cond }\rangle_{V}::= & x=y\left|R\left(z_{1}, \ldots, z_{r}\right)\right|\langle\text { cond }\rangle_{V} \vee\langle\text { cond }\rangle_{V} \mid \neg\langle\text { cond }\rangle_{V}
\end{aligned}
$$

Without loss of generality, we can assume that our programs do not use relations (they can be modeled with functions) and that every condition is either an equality or disequality between variables (arbitrary Boolean combinations can be modeled with nested if-then-else). When the set of variables $V$ is clear from context, we will omit the subscript $V$ from $\langle s t m t\rangle_{V}$ and $\langle\text { cond }\rangle_{V}$.

Program Executions. An execution over $V$ is a finite word over the alphabet

$$
\begin{aligned}
\Pi_{V}=\{" x:=y ", " x:=f(\bar{z}) " & , \operatorname{assume}(x=y) ", \text { "assume }(x \neq y) ", \\
& \text { "assert } \left.(\perp) " \mid x, y \in V, \bar{z} \in V^{r}, f \in \mathcal{F}\right\} .
\end{aligned}
$$

The set of complete executions for a program $p$ over $V$, denoted $\operatorname{Exec}(p)$, is a regular language. See [30] for a straightforward definition. The set PExec $(p)$ of partial executions is the set of prefixes of complete executions in $\operatorname{Exec}(p)$. We refer to partial executions as simply executions, and clarify as needed when the distinction is important. 
Semantics. The semantics of executions is given in terms of data models. A data model $\mathcal{M}=(U, \mathcal{I})$ is a first order structure over $\Sigma$ comprised of a universe $U$ and an interpretation function $\mathcal{I}$ for the program symbols. The semantics of an execution $\pi$ over a data model $\mathcal{M}$ is given by a configuration $\sigma(\pi, \mathcal{M}): V \rightarrow U$ which maps each variable to its value in the universe $U$ at the end of $\pi$. This notion is straightforward and we skip the formal definition (see [39] for details). For a fixed program $p$, any particular data model corresponds to at most one complete execution $\pi \in \operatorname{Exec}(p)$.

An execution $\pi$ is feasible in a data model $\mathcal{M}$ if for every prefix $\rho=\rho^{\prime}$. $\operatorname{assume}(x \sim y)$ of $\pi$ (where $\sim \in\{=, \neq\}$ ), we have $\sigma\left(\rho^{\prime}, \mathcal{M}\right)(x) \sim \sigma\left(\rho^{\prime}, \mathcal{M}\right)(y)$. Execution $\pi$ is said to be correct in a data model $\mathcal{M}$ if for every prefix of $\pi$ of the form $\rho=\rho^{\prime} \cdot \operatorname{assert}(\perp)$, we have that $\rho^{\prime}$ is not feasible, or infeasible in $\mathcal{M}$. Finally, a program $p$ is said to be correct if for all data models $\mathcal{M}$ and executions $\pi \in \operatorname{PExec}(p), \pi$ is correct in $\mathcal{M}$.

\subsection{The Program Synthesis Problem}

We are now ready to define the program synthesis problem. Our approach will be to allow users to specify a grammar and ask for the synthesis of a program from the grammar. We allow the user to express specifications using assertions in the program to be synthesized.

Grammar Schema and Input Grammar. In our problem formulation, we allow users to define a grammar which conforms to a schema, given below. The input grammars allow the usual context-free power required to describe proper nesting/bracketing of program expressions, but disallow other uses of the context-free power, such as counting statements.

For example, we disallow the grammar in Fig. 4. This grammar has two non-terminals $S$ (the start symbol) and $T$. It generates programs with a conditional that has the same number of assignments in the if and else branches. We assume a countably infinite set $P N$ of nonterminals and a countably infinite set $P V$ of program variables. The grammar schema $\mathcal{S}$ over $P N$ and

$$
\begin{aligned}
S \rightarrow & \text { if }(\mathrm{x}=\mathrm{y}) \\
& \text { then } \mathrm{u}:=\mathrm{v} T \mathrm{u}:=\mathrm{v} \\
T \rightarrow & \text { else } \\
T \rightarrow & ; \mathrm{u}:=\mathrm{v} \quad T \quad \mathrm{u}:=\mathrm{v} ;
\end{aligned}
$$

Fig. 4. Grammar with counting $P V$ is an infinite collection of productions:

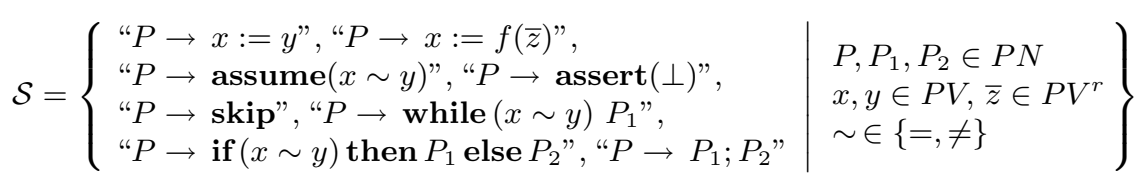

An input grammar $\mathcal{G}$ is any finite subset of the schema $\mathcal{S}$, and it defines a set of programs, denoted $L(\mathcal{G})$. We can now define the main problem addressed in this work. 
Definition 1 (Uninterpreted Program Realizability and Synthesis). Given an input grammar $\mathcal{G}$, the realizability problem is to determine whether there is an uninterpreted program $p \in L(\mathcal{G})$ such that $p$ is correct. The synthesis problem is to determine the above, and further, if realizable, synthesize a correct program $p \in L(\mathcal{G})$.

Example 6. Consider the program with a hole from Example 1 (Fig. 1, left). We can model that synthesis problem in our framework with the following grammar.

$$
\begin{aligned}
& S \rightarrow P_{1} ; P_{2} ; P_{\langle\langle ? ?\rangle\rangle ; P_{3}} \\
& P_{1} \rightarrow \text { "cipher }:=\text { enc (secret, key)" } \quad P_{3} \rightarrow \text { "assert(z = secret)" } \\
& P_{2} \rightarrow \text { "assume }(\text { secret }=\operatorname{dec}(\text { cipher, key })) "
\end{aligned}
$$

Here, $V_{\langle\langle ? ?\rangle\rangle}=\left\{\right.$ cipher, $\left.\mathrm{y}_{1}, \ldots, \mathrm{y}_{n}\right\}$ and the grammar $\langle s t m t\rangle_{\langle\langle ? ?\rangle\rangle}$ is that of Sect. 3, restricted to loop-free programs. Any program generated from this grammar indeed matches the template from Fig. 1 (left) and any such program is correct if it satisfies the last assertion for all models, i.e., all interpretations of the function symbols enc and dec and for all initial values of the variables in $V=V_{\langle\langle ? ?\rangle\rangle} \cup\{$ key, secret $\}$.

\section{Undecidability of Uninterpreted Program Synthesis}

Since verification of uninterpreted programs with loops is undecidable [39,42], the following is immediate.

Theorem 1. The uninterpreted program synthesis problem is undecidable.

We next consider synthesizing loop-free uninterpreted programs (for which verification reduces to satisfiability of quantifier-free EUF) from grammars conforming to the following schema:

$\mathcal{S}_{\text {loop-free }}=\mathcal{S} \backslash\left\{\right.$ "P $\rightarrow$ while $\left.(x \sim y) P_{1} " \mid P, P_{1} \in P N, x, y \in P V, \sim \in\{=, \neq\}\right\}$

Theorem 2. The uninterpreted program synthesis problem is undecidable for the schema $\mathcal{S}_{\text {loop-free. }}$.

This is a corollary of the following stronger result: synthesis of straight-line uninterpreted programs (conforming to schema $\mathcal{S}_{\mathrm{SLP}}$ below) is undecidable.

$$
\begin{array}{r}
\mathcal{S}_{\mathrm{SLP}}=\mathcal{S}_{\text {loop-free }} \backslash\left\{\text { "P } \rightarrow \operatorname{if}(x \sim y) \text { then } P_{1} \text { else } P_{2} " \mid P, P_{1}, P_{2} \in P N,\right. \\
x, y \in P V, \sim \in\{=, \neq\}\}
\end{array}
$$

Theorem 3. The uninterpreted program synthesis problem is undecidable for the schema $\mathcal{S}_{S L P}$.

In summary, program synthesis of even straight-line uninterpreted programs, which have neither conditionals nor iteration, is already undecidable. The notion of coherence for uninterpreted programs was shown to yield decidable verification in [39]. As we'll see in Sect. 5, restricting to coherent programs yields decidable synthesis, even for programs with conditionals and iteration. 


\section{Synthesis of Coherent Uninterpreted Programs}

In this section, we present the main result of the paper: grammar-restricted program synthesis for uninterpreted coherent programs [39] is decidable. Intuitively, coherence allows us to maintain congruence closure in a streaming fashion when reading a coherent execution. First we recall the definition of coherent executions and programs in Sect.5.1 and also the algorithm for verification of such programs. Then we introduce the synthesis procedure, which works by constructing a two-way alternating tree automaton. We briefly discuss this class of tree automata in Sect. 5.2 and recall some standard results. In Sects. 5.3, 5.4 and 5.5 we describe the details of the synthesis procedure, argue its correctness, and discuss its complexity. In Sect.5.6, we present a tight lower bound result.

\subsection{Coherent Executions and Programs}

The notion of coherence for an execution $\pi$ is defined with respect to the terms it computes. Intuitively, at the beginning of an execution, each variable $x \in V$ stores some constant term $\widehat{x} \in \mathcal{C}$. As the execution proceeds, new terms are computed and stored in variables. Let Terms $s_{\Sigma}$ be the set of all ground terms defined using the constants and functions in $\Sigma$. Formally, the term corresponding to a variable $x \in V$ at the end of an execution $\pi \in \Pi_{V}^{*}$, denoted $\mathrm{T}(\pi, x) \in$ Terms $_{\Sigma}$, is inductively defined as follows. We assume that the set of constants $\mathcal{C}$ includes a designated set of initial constants $\widehat{V}=\{\widehat{x} \mid x \in V\} \subseteq \mathcal{C}$.

$$
\begin{aligned}
& \mathrm{T}(\varepsilon, x)=\widehat{x} \\
& \mathrm{~T}(\pi \cdot " x:=y ", x)=\mathrm{T}(\pi, y) \quad x, y \in V \\
& \mathrm{~T}\left(\pi \cdot " x:=f\left(z_{1}, \ldots, z_{r}\right) ", x\right)=f\left(\mathrm{~T}\left(\pi, z_{1}\right), \ldots, \mathrm{T}\left(\pi, z_{r}\right)\right) x, z_{1}, \ldots, z_{r} \in V \\
& \mathrm{~T}(\pi \cdot a, x)=\mathrm{T}(\pi, x) \quad \text { otherwise }
\end{aligned}
$$

We will use $\mathrm{T}(\pi)$ to denote the set $\left\{\mathrm{T}\left(\pi^{\prime}, x\right) \mid x \in V, \pi^{\prime}\right.$ is a prefix of $\left.\pi\right\}$.

A related notion is the set of term equality assumptions that an execution accumulates, which we formalize as $\alpha: \pi \rightarrow \mathcal{P}\left(\operatorname{Terms}_{\Sigma} \times\right.$ Terms $\left._{\Sigma}\right)$, and define inductively as $\alpha(\varepsilon)=\varnothing, \alpha(\pi \cdot$ "assume $(x=y)$ " $)=\alpha(\pi) \cup\{(\mathrm{T}(\pi, x), \mathrm{T}(\pi, y))\}$, and $\alpha(\pi \cdot a)=\alpha(\pi)$ otherwise.

For a set of term equalities $A \subseteq$ Terms $_{\Sigma} \times$ Terms $_{\Sigma}$, and two ground terms $t_{1}, t_{2} \in$ Terms $_{\Sigma}$, we say $t_{1}$ and $t_{2}$ are equivalent modulo $A$, denoted $t_{1} \cong_{A} t_{2}$, if $A \models t_{1}=t_{2}$. For a set of terms $S \subseteq$ Terms $_{\Sigma}$, and a term $t \in$ Terms $_{\Sigma}$ we write $t \in_{A} S$ if there is a term $t^{\prime} \in S$ such that $t \cong_{A} t^{\prime}$. For terms $t, s \in \operatorname{Terms}_{\Sigma}$, we say $s$ is a superterm modulo $A$ of $t$, denoted $t \preccurlyeq A s$ if there are terms $t^{\prime}, s^{\prime} \in$ Terms $_{\Sigma}$ such that $t \cong_{A} t^{\prime}, s \cong_{A} s^{\prime}$ and $s^{\prime}$ is a superterm of $t^{\prime}$.

With the above notation in mind, we now review the notion of coherence.

Definition 2 (Coherent Executions and Programs [39]). An execution $\pi \in$ $\Pi_{V}^{*}$ is said to be coherent if it satisfies the following two conditions.

Memoizing. Let $\rho=\rho^{\prime} \cdot " x:=f(\bar{y})$ " be a prefix of $\pi$. If $t_{x}=T(\rho, x) \in_{\alpha\left(\rho^{\prime}\right)} T\left(\rho^{\prime}\right)$, then there is a variable $z \in V$ such that $t_{x} \cong_{\alpha\left(\rho^{\prime}\right)} t_{z}$, where $t_{z}=T\left(\rho^{\prime}, z\right)$. 
Early Assumes. Let $\rho=\rho^{\prime}$. "assume $(x=y)$ " be a prefix of $\pi, t_{x}=T\left(\rho^{\prime}, x\right)$ and $t_{y}=T\left(\rho^{\prime}, y\right)$. If there is a term $s \in T\left(\rho^{\prime}\right)$ such that either $t_{x} \preccurlyeq \alpha\left(\rho^{\prime}\right) s$ or $t_{y} \preccurlyeq \alpha\left(\rho^{\prime}\right) s$, then there is a variable $z \in V$ such that $s \cong_{\alpha\left(\rho^{\prime}\right)} t_{z}$, where $t_{z}=T\left(\rho^{\prime}, z\right)$.

A program $p$ is coherent if every complete execution $\pi \in \operatorname{Exec}(p)$ is coherent.

The following theorems due to [39] establish the decidability of verifying coherent programs and also of checking if a program is coherent.

Theorem 4 ([39]). The verification problem for coherent programs, i.e. checking if a given uninterpreted coherent program is correct, is decidable.

Theorem 5 ([39]). The problem of checking coherence, i.e. checking if a given uninterpreted program is coherent, is decidable.

The techniques used in [39] are automata theoretic. They allow us to construct an automaton $\mathcal{A}_{\text {exec }}{ }^{2}$, of size $O\left(2^{\text {poly }(|V|)}\right)$, which accepts all coherent executions that are also correct.

To give some intuition for the notion of coherence, we illustrate simple example programs that are not coherent. Consider program $p_{0}$ below, which is not coherent because it fails to be memoizing.

$$
p_{0} \triangleq \quad \mathrm{x}:=\mathrm{f}(\mathrm{y}) ; \mathrm{x}:=\mathrm{f}(\mathrm{x}) ; \mathrm{z}:=\mathrm{f}(\mathrm{y})
$$

The first and third statements compute $f(\widehat{y})$, storing it in variables $x$ and $z$, respectively, but the term is dropped after the second statement and hence is not contained in any program variable when the third statement executes. Next consider program $p_{1}$, which is not coherent because it fails to have early assumes.

$$
p_{1} \triangleq \mathrm{x}:=\mathrm{f}(\mathrm{w}) ; \mathrm{x}:=\mathrm{f}(\mathrm{x}) ; \mathrm{y}:=\mathrm{f}(\mathrm{z}) ; \mathrm{y}:=\mathrm{f}(\mathrm{y}) ; \operatorname{assume}(\mathrm{w}=\mathrm{z})
$$

Indeed, the assume statement is not early because superterms of $w$ and $z$, namely $f(\widehat{w})$ and $f(\widehat{z})$, were computed and subsequently dropped before the assume.

Intuitively, the coherence conditions are necessary to allow equality information to be tracked with finite memory. We can make this stark by tweaking the example for $p_{1}$ above as follows.

$$
\begin{aligned}
& p_{1}^{\prime} \triangleq \quad \mathrm{x}:=\mathrm{f}(\mathrm{w}) ; \underbrace{\mathrm{x}:=\mathrm{f}(\mathrm{x}) \cdots \mathrm{x}:=\mathrm{f}(\mathrm{x})}_{n \text { times }} ; \\
& \mathrm{y}:=\mathrm{f}(\mathrm{z}) ; \underbrace{\mathrm{y}:=\mathrm{f}(\mathrm{y}) \cdots \mathrm{y}:=\mathrm{f}(\mathrm{y})}_{n \text { times }} ; \operatorname{assume}(\mathrm{w}=\mathrm{z})
\end{aligned}
$$

Observe that, for large $n$ (e.g. $n>100$ ), many terms are computed and dropped by this program, like $f^{42}(\widehat{x})$ and $f^{99}(\widehat{y})$ for instance. The difficulty with this

\footnotetext{
${ }^{2}$ We use superscripts '... ' and ' $\mathrm{s}$ ' for word and tree automata, respectively.
} 
program, from a verification perspective, is that the assume statement entails equalities between many terms which have not been kept track of. Imagine trying to verify the following program

$$
p_{2} \triangleq p_{1}^{\prime} ; \operatorname{assert}(\mathrm{x}=\mathrm{y})
$$

Let $\pi_{p_{1}^{\prime}} \in \operatorname{Exec}\left(p_{1}^{\prime}\right)$ be the unique complete execution of $p_{1}^{\prime}$. If we examine the details, we see that $t_{x}=\mathrm{T}\left(\pi_{p_{1}^{\prime}}, x\right)=f^{101}(\widehat{w})$ and $t_{y}=\mathrm{T}\left(\pi_{p_{1}^{\prime}}, y\right)=f^{101}(\widehat{z})$. The assertion indeed holds because $t_{x} \cong_{\{(\widehat{w}, \widehat{z})\}} t_{y}$. However, to keep track of this fact requires remembering an arbitrary number of terms that grows with the size of the program. Finally, we note that the coherence restriction is met by many single-pass algorithms, e.g. searching and manipulation of lists and trees.

\subsection{Overview of the Synthesis Procedure}

Our synthesis procedure uses tree automata. We consider tree representations of programs, or program trees. The synthesis problem is thus to check if there is a program tree whose corresponding program is coherent, correct, and belongs to the input grammar $\mathcal{G}$.

The synthesis procedure works as follows. We first construct a top-down tree automaton $\mathcal{A}_{\mathcal{G}}$ that accepts the set of trees corresponding to the programs generated by $\mathcal{G}$. We next construct another tree automaton $\mathcal{A}_{\mathrm{cc}}$, which accepts all trees corresponding to programs that are coherent and correct. $\mathcal{A}_{\mathrm{cc}}^{\mathrm{i}}$ is a two-way alternating tree automaton that simulates all executions of an input program tree and checks that each is both correct and coherent. In order to simulate longer and longer executions arising from constructs like while-loops, the automaton traverses the input tree and performs multiple passes over subtrees, visiting the internal nodes of the tree many times. We then translate the two-way alternating tree automaton to an equivalent (one-way) nondeterministic top-down tree automaton by adapting results from $[33,53]$ to our setting. Finally, we check emptiness of the intersection between this top-down automaton and the grammar automaton $\mathcal{A}_{\mathcal{G}}$. The definitions for trees and the relevant automata are standard, and we refer the reader to [14] and to our technical report [30].

\subsection{Tree Automaton for Program Trees}

Every program can be represented as a tree whose leaves are labeled with basic statements like " $x:=y$ " and whose internal nodes are labeled with constructs like while and seq (an alias for the sequencing construct ';'), which have subprograms as children. Essentially, we represent the set of programs generated by an input grammar $\mathcal{G}$ as a regular set of program trees, accepted by a nondeterministic top-down tree automaton $\mathcal{A}_{\mathcal{G}}$. The construction of $\mathcal{A}_{\mathcal{G}}$ mimics the standard construction for tree automata that accept parse trees of context free grammars. The formalization of this intuition is straightforward, and we refer the reader to [30] for details. We note the following fact regarding the construction of the acceptor of program trees from a particular grammar $\mathcal{G}$.

Lemma 1. $\mathcal{A}_{\mathcal{G}}^{\hat{B}}$ has size $O(|\mathcal{G}|)$ and can be constructed in time $O(|\mathcal{G}|)$. 


\subsection{Tree Automaton for Simulating Executions}

We now discuss the construction of the two-way alternating tree automaton $\mathcal{A}_{\text {cc }}^{\hat{n}}$ that underlies our synthesis procedure. A two-way alternating tree automaton consists of a finite set of states and a transition function that maps tuples $(q, m, a)$ of state, incoming direction, and node labels to positive Boolean formulas over pairs $\left(q^{\prime}, m^{\prime}\right)$ of next state and next direction. In the case of our binary program trees, incoming directions come from $\left\{D, U_{L}, U_{R}\right\}$, corresponding to coming down from a parent, and up from left and right children. Next directions come from $\{U, L, R\}$, corresponding to going up to a parent, and down to left and right children.

The automaton $\mathcal{A}_{\mathrm{cc}}$ is designed to accept the set of all program trees that correspond to correct and coherent programs. This is achieved by ensuring that a program tree is accepted precisely when all executions of the program it represents are accepted by the word automaton $\mathcal{A}_{\text {exec }}^{\cdots}$ (Sect. 5.1). The basic idea behind $\mathcal{A}_{\mathrm{cc}}^{*}$ is as follows. Given a program tree $T$ as input, $\mathcal{A}_{\mathrm{cc}}^{*}$ traverses $T$ and explores all the executions of the associated program. For each execution $\sigma, \mathcal{A}_{\mathrm{cc}}$ keeps track of the state that the word automaton $\mathcal{A}_{\text {exec }}^{-}$would reach after reading $\sigma$. Intuitively, an accepting run of $\mathcal{A}_{\text {cc }}^{*}$ is one which never visits the unique rejecting state of $\mathcal{A}_{\text {exec }}^{-\cdots}$ during simulation.

We now give the formal description of $\mathcal{A}_{\mathrm{cc}}=\left(Q^{\mathrm{cc}}, I^{\mathrm{cc}}, \delta_{0}^{\mathrm{cc}}, \delta_{1}^{\mathrm{cc}}, \delta_{2}^{\mathrm{cc}}\right)$, which works over the alphabet $\Gamma_{V}$ described in Sect. 5.3.

States. Both the full set of states and the initial set of states for $\mathcal{A}_{\text {cc }}$ coincide with those of the word automaton $\mathcal{A}_{\text {exec }}^{-}$. That is, $Q^{\mathrm{cc}}=Q^{\text {exec }}$ and $I^{\mathrm{cc}}=\left\{q_{0}^{\text {exec }}\right\}$, where $q_{0}^{\text {exec }}$ is the unique starting state of $\mathcal{A}_{\text {exec }}^{\cdots}$.

Transitions. For intuition, consider the case when the automaton's control is in state $q$ reading an internal tree node $n$ with one child and which is labeled by $a=$ "while $(x=y)$ ". In the next step, the automaton simultaneously performs two transitions corresponding to two possibilities: entering the loop after assuming the guard " $x=y$ " to be true and exiting the loop with the guard being false. In the first of these simultaneous transitions, the automaton moves to the left child $n \cdot L$, and its state changes to $q_{1}^{\prime}$, where $q_{1}^{\prime}=\delta^{\operatorname{exec}}(q$, "assume $(x=y) ")$. In the second simultaneous transition, the automaton moves to the parent node $n \cdot U$ (searching for the next statement to execute, which follows the end of the loop) and changes its state to $q_{2}^{\prime}$, where $q_{2}^{\prime}=\delta^{\text {exec }}(q$, "assume $(x \neq y)$ "). We encode these two possibilities as a conjunctive transition of the two-way alternating automaton. That is, $\delta_{1}^{\mathrm{cc}}(q, m, a)=\left(\left(q_{1}^{\prime}, L\right) \wedge\left(q_{2}^{\prime}, U\right)\right)$.

For every $i, m, a$, we have $\delta_{i}\left(q_{\text {reject }}, m, a\right)=\perp$, where $q_{\text {reject }}$ is the unique, absorbing rejecting state of $\mathcal{A}_{\text {exec }}^{-}$. Below we describe the transitions from all other states $q \neq q_{\text {reject }}$. All transitions $\delta_{i}(q, m, a)$ not described below are $\perp$.

Transitions from the Root. At the root node, labeled by "root", the automaton transitions as follows:

$$
\delta_{1}^{\mathrm{cc}}(q, m, \text { root })= \begin{cases}(q, L) & \text { if } m=D \\ \text { true } & \text { otherwise }\end{cases}
$$


A two-way tree automaton starts in the configuration where $m$ is set to $D$. This means that in the very first step the automaton moves to the child node (direction $L$ ). If the automaton visits the root node in a subsequent step (marking the completion of an execution), then all transitions are enabled.

Transitions from Leaf Nodes. For a leaf node with label $a \in \Gamma_{0}$ and state $q$, the transition of the automaton is $\delta_{0}^{\mathrm{cc}}(q, D, a)=\left(\delta^{\operatorname{exec}}(q, a), U\right)$. That is, when the automaton visits a leaf node from the parent, it simulates reading $a$ in $\mathcal{A}_{\text {exec }}^{-\cdots}$ and moves to the resulting state in the parent node.

Transitions from "while" Nodes. As described earlier, when reading a node labeled by "while $(x \sim y)$ ", where $\sim \in\{=, \neq\}$, the automaton simulates both the possibility of entering the loop body as well as the possibility of exiting the loop. This corresponds to a conjunctive transition:

$$
\begin{aligned}
\delta_{1}^{\mathrm{cc}}(q, m, \text { while }(x \sim y) ") & =\left(q^{\prime}, L\right) \wedge\left(q^{\prime \prime}, U\right) \\
\text { where } q^{\prime} & =\delta^{\operatorname{exec}}(q, " \operatorname{assume}(x \sim y) ") \\
\text { and } q^{\prime \prime} & =\delta^{\operatorname{exec}}(q, \text { "assume }(x \not y) ")
\end{aligned}
$$

Above, $\neq$ refers to " $="$ when $\sim$ is " $\neq "$, and vice versa. The first conjunct corresponds to the execution where the program enters the loop body (assuming the guard is true), and thus control moves to the left child of the current node, which corresponds to the loop body. The second conjunct corresponds to the execution where the loop guard is false and the automaton moves to the parent of the current tree node. Notice that, in both the conjuncts above, the direction in which the tree automaton moves does not depend on the last move $m$ of the state. That is, no matter how the program arrives at a while statement, the automaton simulates both the possibilities of entering or exiting the loop body.

Transitions from "ite" Nodes. At a node labeled "ite $(x \sim y)$ ", when coming down the tree from the parent, the automaton simulates both branches of the conditional:

$$
\begin{aligned}
\delta_{2}^{\text {cc }}(q, D, " i \operatorname{ite}(x \sim y) ") & =\left(q^{\prime}, L\right) \wedge\left(q^{\prime \prime}, R\right) \\
\text { where } q^{\prime} & =\delta^{\operatorname{exec}}(q, " \operatorname{assume}(x \sim y) ") \\
\text { and } q^{\prime \prime} & =\delta^{\operatorname{exec}}(q, " \operatorname{assume}(x \not y) ")
\end{aligned}
$$

The first conjunct in the transition corresponds to simulating the word automaton on the condition $x \sim y$ and moving to the left child, i.e. the body of the then branch. Similarly, the second conjunct corresponds to simulating the word automaton on the negation of the condition and moving to the right child, i.e. the body of the else branch. 
Now consider the case when the automaton moves up to an ite node from a child node. In this case, the automaton moves up to the parent node (having completed simulation of the then or else branch) and the state $q$ remains unchanged:

$$
\delta_{2}^{\mathrm{cc}}(q, m, \text { "ite }(x \sim y) ")=(q, U) \quad m \in\left\{U_{L}, U_{R}\right\}
$$

Transitions from "seq" Nodes. In this case, the automaton moves either to the left child, the right child, or to the parent, depending on the last move. It does not change the state component. Formally,

$$
\delta_{2}^{\mathrm{cc}}(q, m, \text { "seq" })=\left\{\begin{array}{l}
(q, L) \text { if } m=D \\
(q, R) \text { if } m=U_{L} \\
(q, U) \text { if } m=U_{R}
\end{array}\right.
$$

The above transitions match the straightforward semantics of sequencing two statements $s_{1} ; s_{2}$. If the automaton visits from the parent node, it next moves to the left child to simulate $s_{1}$. When it finishes simulating $s_{1}$, it comes up from the left child and enters the right child to begin simulating $s_{2}$. Finally, when simulation of $s_{2}$ is complete, the automaton moves to the parent node, exiting the subtree.

The following lemma asserts the correctness of the automaton construction and states its complexity.

Lemma 2. $\mathcal{A}_{c c}$ accepts the set of all program trees corresponding to correct, coherent programs. It has size $\left|\mathcal{A}_{c c}\right|=O\left(2^{\text {poly( }|V|)}\right)$, and can be constructed in $O\left(2^{\text {poly }(|V|)}\right)$ time.

\subsection{Synthesis Procedure}

The rest of the synthesis procedure goes as follows. We first construct a nondeterministic top-down tree automaton $\mathcal{A}_{\text {cc-td }}^{*}$ such that $L\left(\mathcal{A}_{\text {cc-td }}^{*}\right)=L\left(\mathcal{A}_{\text {cc }}^{*}\right)$. An adaptation of results from $[33,53]$ ensures that $\mathcal{A}_{\text {cc-td }}$ has size $\left|\mathcal{A}_{\text {cc-td }}\right|=O\left(2^{2^{\text {poly }}} \mid\right.$ and can be constructed in time $O\left(2^{2^{\text {poly(|V|) }}}\right)$. Next we construct a top-down nondeterministic tree automaton $\mathcal{A}^{\star}$ such that $L\left(\mathcal{A}^{\star}\right)=L\left(\mathcal{A}_{\text {cc-td }}^{*}\right) \cap L\left(\mathcal{A}_{\mathcal{G}}\right)=$

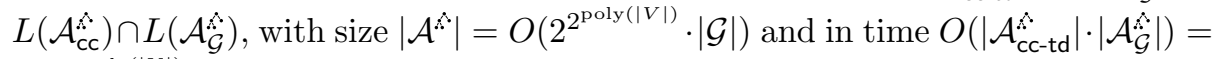

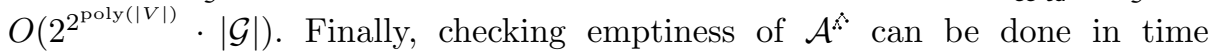
$O\left(\left|\mathcal{A}^{\hat{k}}\right|\right)=O\left(2^{2^{\text {poly }(|V|)}} \cdot|\mathcal{G}|\right)$. If non-empty, a program tree can be constructed.

This gives us the central upper bound result of the paper.

Theorem 6. The grammar-restricted synthesis problem for uninterpreted coherent programs is decidable in 2EXPTIME, and in particular, in time doubly exponential in the number of variables and linear in the size of the input grammar. Furthermore, a tree automaton representing the set of all correct coherent programs that conform to the grammar can be constructed in the same time. 


\subsection{Matching Lower Bound}

Our synthesis procedure is optimal. We prove a 2EXPTIME lower bound for the synthesis problem by reduction from the 2EXPTIME-hard acceptance problem of alternating Turing machines (ATMs) with exponential space bound [12]. Full details of the reduction can be found in [30].

Theorem 7. The grammar-restricted synthesis problem for coherent uninterpreted programs is 2EXPTIME-hard.

\section{Further Results}

In this section, we give results for variants of uninterpreted program synthesis in terms of transition systems, Boolean programs, and recursive programs.

\subsection{Synthesizing Transition Systems}

Here, rather than synthesizing programs from grammars, we consider instead the synthesis of transition systems whose executions must belong to a regular set. Our main result is that the synthesis problem in this case is EXPTIME-complete, in contrast to grammar-restricted program synthesis which is 2EXPTIMEcomplete.

Transition System Definition and Semantics. Let us fix a set of program variables $V$ as before. We consider the following finite alphabet

$\Sigma_{V}=\left\{" x:=y ", " x:=f(\bar{z}) ", " \operatorname{assert}(\perp) ", " \operatorname{check}(x=y) " \mid x, y, \in V, \bar{z} \in V^{r}\right\}$

Let us define $\Gamma_{V} \subseteq \Sigma_{V}$ to be the set of all elements of the form "check $(x=y)$ ", where $x, y \in V$. We refer to the elements of $\Gamma_{V}$ as check letters.

A (deterministic) transition system $T S$ over $V$ is a tuple $\left(Q, q_{0}, H, \lambda, \delta\right)$, where $Q$ is a finite set of states, $q_{0} \in Q$ is the initial state, $H \subseteq Q$ is the set of halting states, $\lambda: Q \rightarrow \Sigma_{V}$ is a labeling function such that for any $q \in Q$, if $\lambda(q)=$ "assert $(\perp)$ " then $q \in H$, and $\delta:(Q \backslash H) \rightarrow Q \cup(Q \times Q)$ is a transition function such that for any $q \in Q \backslash H, \delta(q) \in Q \times Q$ iff $\lambda(q) \in \Gamma_{V}$.

We define the semantics of a transition system using the set of executions that it generates. A (partial) execution $\pi$ of a transition system $T S=\left(Q, q_{0}, H, \lambda, \delta\right)$ over variables $V$ is a finite word over the induced execution alphabet $\Pi_{V}$ (from Sect.3) with the following property. If $\pi=a_{0} a_{1} \ldots a_{n}$ with $n \geq 0$, then there exists a sequence of states $q_{j_{0}}, q_{j_{1}}, \ldots, q_{j_{n}}$ with $q_{j_{0}}=q_{0}$ such that $(0 \leq i \leq n)$ :

- If $\lambda\left(q_{j_{i}}\right) \notin \Gamma_{V}$ then $a_{i}=\lambda\left(q_{j_{i}}\right)$, and if $i<n$ then $q_{j_{i+1}}=\delta\left(q_{j_{i}}\right)$.

- Otherwise $\begin{cases}\text { either } & a_{i}=" \operatorname{assume}(x=y) " \text { and } i<n \Rightarrow q_{j_{i+1}}=\delta\left(q_{j_{i}}\right) L_{1}, \\ \text { or } & a_{i}=" \operatorname{assume}(x \neq y) " \text { and } i<n \Rightarrow q_{j_{i+1}}=\delta\left(q_{j_{i}}\right) L_{2}\end{cases}$ 
In the above, we denote pair projection with $l$, i.e., $\left(t_{1}, t_{2}\right) l_{i}=t_{i}$, where $i \in\{1,2\}$. A complete execution is an execution whose corresponding final state $\left(q_{n}\right.$ above $)$ is in $H$. For any transition system $T S$, we denote the set of its executions by $\operatorname{Exec}(T S)$ and the set of its complete executions by CompExec $(T S)$. The notions of correctness and coherence for transition systems are identical to their counterparts for programs.

The Transition System Synthesis Problem. We consider transition system specifications that place restrictions on executions (both partial and complete) using two regular languages $S$ and $R$. Executions must belong to the first language $S$ (which is prefix-closed) and all complete executions must belong to the second language $R$. A specification is given as two deterministic automata $\mathcal{A}_{S}$ and $\mathcal{A}_{R}$ over executions, where $L\left(\mathcal{A}_{S}\right)=S$ and $L\left(\mathcal{A}_{R}\right)=R$. For a transition system $T S$ and specification automata $\mathcal{A}_{S}$ and $\mathcal{A}_{R}$, whenever $\operatorname{Exec}(T S) \subseteq L\left(\mathcal{A}_{S}\right)$ and CompExec $(T S) \subseteq L\left(\mathcal{A}_{R}\right)$ we say that $T S$ satisfies its (syntactic) specification. Note that this need not entail correctness of $T S$. Splitting the specification into partial executions $S$ and complete executions $R$ allows us, among other things, to constrain the executions of non-halting transition systems.

Definition 3 (Transition System Realizability and Synthesis). Given a finite set of program variables $V$ and deterministic specification automata $\mathcal{A}_{S}$ (prefix-closed) and $\mathcal{A}_{R}$ over the execution alphabet $\Pi_{V}$, decide if there is a correct, coherent transition system TS over $V$ that satisfies the specification. Furthermore, produce one if it exists.

Since programs are readily translated to transition systems (of similar size), the transition system synthesis problem seems, at first glance, to be a problem that ought to have similar complexity. However, as we show, it is crucially different in that it allows the synthesized transition system to have complete information of past commands executed at any point. We will observe in this section that the transition system synthesis problem is EXPTIME-complete.

To see the difference between program and transition system synthesis, consider program skeleton $P$ from Example 2 in Sect. 2. The problem is to fill the hole in $P$ with either y $:=\mathrm{T}$ or $\mathrm{y}:=\mathrm{F}$. Observe that when $P$ executes, there are two different executions that lead to the hole. In grammar-restricted program synthesis, the hole must be filled by a sub-program that is executed no matter how the hole is reached, and hence no such program exists. However, when we model this problem in the setting of transition systems, the synthesizer is able to produce transitions that depend on how the hole is reached. In other words, it does not fill the hole in $P$ with uniform code. In this sense, in grammar-restricted program synthesis, programs have incomplete information of the past. We crucially exploited this difference in the proof of 2EXPTIME-hardness for grammarrestricted program synthesis (see [30]). No such incomplete information can be enforced by regular execution specifications in transition system synthesis, and indeed the problem turns out to be easier: transition system realizability and synthesis are EXPTIME-complete. 
Theorem 8. Transition system realizability is decidable in time exponential in the number of program variables and polynomial in the size of the automata $\mathcal{A}_{S}$ and $\mathcal{A}_{R}$. Furthermore, the problem is EXPTIME-complete. When realizable, within the same time bounds we can construct a correct, coherent transition system whose partial and complete executions are in $L\left(\mathcal{A}_{S}^{-}\right)$and $L\left(\mathcal{A}_{R}^{-}\right)$, respectively.

\subsection{Synthesizing Boolean Programs}

Here we observe corollaries of our results when applied to the more restricted problem of synthesizing Boolean programs.

In Boolean program synthesis we interpret variables in programs over the Boolean domain $\{T, F\}$, and we disallow computations of uninterpreted functions and the checking of uninterpreted relations. Standard Boolean functions like $\wedge$ and $\neg$ are instead allowed, but note that these can be modeled using conditional statements. We allow for nondeterminism with a special assignment " $b:=*$ ", which assigns $b$ nondeterministically to $T$ or $F$. As usual, a program is correct when it satisfies all its assertions.

Synthesis of Boolean programs can be reduced to uninterpreted program synthesis using two special constants $T$ and $F$. Each nondeterministic assignment is modeled by computing a next function on successive nodes of a linked list, accessing a nondeterministic value by computing key on the current node, and assuming the result is either $T$ or $F$. Since uninterpreted programs must satisfy assertions in all models, this indeed captures nondeterministic assignment. Further, every term ever computed in such a program is equivalent to $T$ or $F$ (by virtue of the interleaved assume statements), making the resulting program coherent. The 2EXPTIME upper bound for Boolean program synthesis now follows from Theorem 6 . We further show that, perhaps surprisingly, the 2EXPTIME lower bound from Sect. 5 can be adapted to prove 2EXPTIMEhardness of Boolean program synthesis.

Theorem 9. The grammar-restricted synthesis problem for Boolean programs is 2EXPTIME-complete, and can be solved in time doubly-exponential in the number of variables and linear in the size of the input grammar.

Thus synthesis for coherent uninterpreted programs is no more complex than Boolean program synthesis, establishing decidability and complexity of a problem which has found wide use in practice - for instance, the synthesis tool SKETCH solves precisely this problem, as it models integers using a small number of bits and allows grammars to restrict programs with holes.

\subsection{Synthesizing Recursive Programs}

We extend the positive result of Sect. 5 to synthesize coherent recursive programs. The setup for the problem is very similar. Given a grammar that identifies a class of recursive programs, the goal is to determine if there is a program in the grammar that is coherent and correct. 
The syntax of recursive programs is similar to the non-recursive case, and we refer the reader to [30] for details. In essence, programs are extended with a new function call construct. Proofs are similar in structure to the non-recursive case, with the added challenge of needing to account for recursive function calls and the fact that $\mathcal{A}_{\text {exec }}^{-\cdots}$ becomes a (visibly) pushdown automaton rather than a standard finite automaton. This gives a 2EXPTIME algorithm for synthesizing recursive programs; a matching lower bound follows from the non-recursive case.

Theorem 10. The grammar-restricted synthesis problem for uninterpreted coherent recursive programs is 2EXPTIME-complete. The algorithm is doubly exponential in the number of program variables and linear in the size of the input grammar. Furthermore, a tree automaton representing the set of all correct, coherent recursive programs that conform to the grammar can be constructed in the same time.

\section{Related Work}

The automata and game-theoretic approaches to synthesis date back to a problem proposed by Church [13], after which a rich theory emerged [9,18,32,48]. The problems considered in this line of work typically deal with a system reacting to an environment interactively using a finite set of signals over an infinite number of rounds. Tree automata over infinite trees, representing strategies, with various infinitary acceptance conditions (Büchi, Rabin, Muller, parity) emerged as a uniform technique to solve such synthesis problems against temporal logic specifications with optimal complexity bounds $[31,38,44,45]$. In this paper, we use an alternative approach from [35] that works on finite program trees, using two-way traversals to simulate iteration. The work in [35], however, uses such representations to solve synthesis problems for programs over a fixed finite set of Boolean variables and against LTL specifications. In this work we use it to synthesize coherent programs that have finitely many variables working over infinite domains endowed with functions and relations.

While decidability results for program synthesis beyond finite data domains are uncommon, we do know of some results of this kind. First, there are decidability results known for synthesis of tranducers with registers [29]. Transducers interactively read a stream of inputs and emit a stream of outputs. Finite-state tranducers can be endowed with a set of registers for storing inputs and doing only equality/disequality comparisons on future inputs. Synthesis of such transducers for temporal logic specifications is known to be decidable. Note that, although the data domain is infinite, there are no functions or relations on data (other than equality), making it a much more restricted class (and grammarbased approaches for syntactically restricting transducers has not been studied). Indeed, with uninterpreted functions and relations, the synthesis problem is undecidable (Theorem 1), with decidability only for coherent programs. In [11], the authors study the problem of synthesizing uninterpreted terms from a grammar that satisfy a first-order specification. They give various decidability and 
undecidability results. In contrast, our results are for programs with conditionals and iteration (but restricted to coherent programs) and for specifications using assertions in code.

Another setting with a decidable synthesis result over unbounded domains is work on strategy synthesis for linear arithmetic satisfiability games [17]. There it is shown that for a satisfiability game, in which two players (SAT and UNSAT) play to prove a formula is satisfiable (where the formula is interpreted over the theory of linear rational arithmetic), if the SAT player has a winning strategy then a strategy can be synthesized. Though the data domain (rationals) is infinite, the game consists of a finite set of interactions and hence has no need for recursion. The authors also consider reachability games where the number of rounds can be unbounded, but present only sound and incomplete results, as checking who wins in such reachability games is undecidable.

Tree automata techniques for accepting finite parse trees of programs was explored in [37] for synthesizing reactive programs with variables over finite domains. In more recent work, automata on finite trees have been explored for synthesizing data completion scripts from input-output examples [55], for accepting programs that are verifiable using abstract interpretations [54], and for relational program synthesis [56].

The work in [36] explores a decidable logic with $\exists^{*} \forall^{*}$ prefixes that can be used to encode synthesis problems with background theories like arithmetic. However, encoding program synthesis in this logic only expresses programs of finite size. Another recent paper [27] explores sound (but incomplete) techniques for showing unrealizability of syntax-guided synthesis problems.

\section{Conclusions}

We presented foundational results on synthesizing coherent programs with uninterpreted functions and relations. To the best of our knowledge, this is the first natural decidable program synthesis problem for programs of arbitrary size which have iteration/recursion, and which work over infinite domains.

The field of program synthesis lacks theoretical results, and especially decidability results. We believe our results to be the first of their kind to fill this lacuna, and we find this paper exciting because it bridges the worlds of program synthesis and the rich classical synthesis frameworks of systems over finite domains using tree automata $[9,18,32,48]$. We believe this link could revitalize both domains with new techniques and applications.

Turning to practical applications of our results, several questions require exploration in future work. First, one might question the utility of programs that verify only with respect to uninterpreted data domains. Recent work [10] has shown that verifying programs using uninterpreted abstractions can be extremely effective in practice for proving programs correct. Also, recent work by Mathur et al. [40] explores ways to add axioms (such as commutativity of functions, axioms regarding partial orders, etc.) and yet preserve decidability of verification. The methods used therein are compatible with our technique, and we 
believe our results can be extended smoothly to their decidable settings. A more elaborate way to bring in complex theories (like arithmetic) would be to marry our technique with the iterative automata-based software verification technique pioneered by work behind the Ultimate tool [23-26]; this won't yield decidable synthesis, but still could result in complete synthesis procedures.

The second concern for practicality is the coherence restriction. There is recent work by Mathur et al. [41] that shows single-pass heap-manipulating programs respect a (suitably adapted) notion of coherence. Adapting our technique to this setting seems feasible, and this would give an interesting application of our work. Finally, it is important to build an implementation of our procedure in a tool that exploits pragmatic techniques for constructing tree automata, and the techniques pursued in [54-56] hold promise.

\section{References}

1. Alur, R., et al.: Syntax-guided synthesis. In: Dependable Software Systems Engineering, NATO Science for Peace and Security Series, D: Information and Communication Security, vol. 40, pp. 1-25. IOS Press (2015)

2. Alur, R., Madhusudan, P.: Visibly pushdown languages. In: Proceedings of the Thirty-sixth Annual ACM Symposium on Theory of Computing, STOC 2004, pp. 202-211. ACM, New York (2004). https://doi.org/10.1145/1007352.1007390

3. Alur, R., Madhusudan, P.: Adding nesting structure to words. J. ACM 56(3), 16:1-16:43 (2009). https://doi.org/10.1145/1516512.1516518

4. Alur, R., Singh, R., Fisman, D., Solar-Lezama, A.: Search-based program synthesis. Commun. ACM 61(12), 84-93 (2018). https://doi.org/10.1145/3208071

5. Bauer-Mengelberg, S.: über die vollständigkeit des logikkalküls. J. Symb. Log. 55(1), 341-342 (1990). https://doi.org/10.2307/2274974

6. Bloem, R., Galler, S.J., Jobstmann, B., Piterman, N., Pnueli, A., Weiglhofer, M.: Specify, compile, run: hardware from PSL. Electr. Notes Theor. Comput. Sci. 190(4), 3-16 (2007). https://doi.org/10.1016/j.entcs.2007.09.004

7. Bloem, R., Jobstmann, B., Piterman, N., Pnueli, A., Sa'ar, Y.: Synthesis of reactive(1) designs. J. Comput. Syst. Sci. 78(3), 911-938 (2012). https://doi.org/10. 1016/j.jcss.2011.08.007

8. Bradley, A.R., Manna, Z.: The Calculus of Computation: Decision Procedures with Applications to Verification. Springer, Heidelberg (2007). https://doi.org/10.1007/ 978-3-540-74113-8

9. Buchi, J.R., Landweber, L.H.: Solving sequential conditions by finite-state strategies. Trans. Am. Math. Soc. 138, 295-311 (1969). https://doi.org/10.2307/1994916

10. Bueno, D., Sakallah, K.A.: euforia: complete software model checking with uninterpreted functions. In: Enea, C., Piskac, R. (eds.) VMCAI 2019. LNCS, vol. 11388, pp. 363-385. Springer, Cham (2019). https://doi.org/10.1007/978-3-03011245-5_17

11. Caulfield, B., Rabe, M.N., Seshia, S.A., Tripakis, S.: What's decidable about syntax-guided synthesis? CoRR abs/1510.08393 (2015)

12. Chandra, A.K., Kozen, D.C., Stockmeyer, L.J.: Alternation. J. ACM 28(1), 114133 (1981). https://doi.org/10.1145/322234.322243

13. Church, A.: Application of recursive arithmetic to the problem of circuit synthesis. Summaries of talks presented at the Summer Institute for Symbolic Logic Cornell University, 1957, 2nd edn., J. Symb. Log. 28(4), 30-50. 3a-45a. (1960) 
14. Comon, H., et al.: Tree automata techniques and applications (2007). https://tata. gforge.inria.fr. Accessed 29 Jun 2020

15. Dolev, D., Yao, A.: On the security of public key protocols. IEEE Trans. Inf. Theory 29(2), 198-208 (1983). https://doi.org/10.1109/TIT.1983.1056650

16. Durgin, N., Lincoln, P., Mitchell, J., Scedrov, A.: Multiset rewriting and the complexity of bounded security protocols. J. Comput. Secur. 12(2), 247-311 (2004). https://doi.org/10.3233/JCS-2004-12203

17. Farzan, A., Kincaid, Z.: Strategy synthesis for linear arithmetic games. PACMPL 2(POPL), 61:1-61:30 (2018). https://doi.org/10.1145/3158149

18. Grädel, E., Thomas, W., Wilke, T. (eds.): Automata, Logics, and Infinite Games: A Guide to Current Research [outcome of a Dagstuhl seminar, February 2001]. Lecture Notes in Computer Science, vol. 2500. Springer, Heidelberg (2002). https:// doi.org/10.1007/3-540-36387-4

19. Gulwani, S.: Automating string processing in spreadsheets using input-output examples. In: POPL, pp. 317-330. ACM (2011). https://doi.org/10.1145/1925844. 1926423

20. Gulwani, S., Harris, W.R., Singh, R.: Spreadsheet data manipulation using examples. Commun. ACM 55(8), 97-105 (2012). https://doi.org/10.1145/2240236. 2240260

21. Gulwani, S., Hernández-Orallo, J., Kitzelmann, E., Muggleton, S.H., Schmid, U., Zorn, B.G.: Inductive programming meets the real world. Commun. ACM 58(11), 90-99 (2015). https://doi.org/10.1145/2736282

22. Gulwani, S., Polozov, O., Singh, R.: Program synthesis. Found. Trends Program. Lang. 4(1-2), 1-119 (2017)

23. Heizmann, M., et al.: Ultimate automizer with smtinterpol. In: Piterman, N., Smolka, S.A. (eds.) TACAS 2013. LNCS, vol. 7795, pp. 641-643. Springer, Berlin Heidelberg, Berlin, Heidelberg (2013). https://doi.org/10.1007/978-3-642-36742$7 \_53$

24. Heizmann, M., Hoenicke, J., Podelski, A.: Refinement of trace abstraction. In: Palsberg, J., Su, Z. (eds.) SAS 2009. LNCS, vol. 5673, pp. 69-85. Springer, Heidelberg (2009). https://doi.org/10.1007/978-3-642-03237-0_7

25. Heizmann, M., Hoenicke, J., Podelski, A.: Nested interpolants. In: Proceedings of the 37th Annual ACM SIGPLAN-SIGACT Symposium on Principles of Programming Languages, POPL 2010, pp. 471-482. ACM, New York (2010). https://doi. org/10.1145/1706299.1706353

26. Heizmann, M., Hoenicke, J., Podelski, A.: Software model checking for people who love automata. In: Sharygina, N., Veith, H. (eds.) CAV 2013. LNCS, vol. 8044, pp. 36-52. Springer, Heidelberg (2013). https://doi.org/10.1007/978-3-642-39799-8_2

27. Hu, Q., Breck, J., Cyphert, J., D'Antoni, L., Reps, T.: Proving unrealizability for syntax-guided synthesis. In: Dillig, I., Tasiran, S. (eds.) CAV 2019. LNCS, vol. 11561, pp. 335-352. Springer, Cham (2019). https://doi.org/10.1007/978-3-03025540-4_18

28. Jha, S., Seshia, S.A.: A theory of formal synthesis via inductive learning. Acta Inf. 54(7), 693-726 (2017). https://doi.org/10.1007/s00236-017-0294-5

29. Khalimov, A., Maderbacher, B., Bloem, R.: Bounded synthesis of register transducers. In: Lahiri, S.K., Wang, C. (eds.) ATVA 2018. LNCS, vol. 11138, pp. 494-510. Springer, Cham (2018). https://doi.org/10.1007/978-3-030-01090-4_29

30. Krogmeier, P., Mathur, U., Murali, A., Madhusudan, P., Viswanathan, M.: Decidable synthesis of programs with uninterpreted functions. CoRR abs/1910.09744 (2019). http://arxiv.org/abs/1910.09744 
31. Kupferman, O., Madhusudan, P., Thiagarajan, P.S., Vardi, M.Y.: Open systems in reactive environments: control and synthesis. In: Palamidessi, C. (ed.) CONCUR 2000. LNCS, vol. 1877, pp. 92-107. Springer, Heidelberg (2000). https://doi.org/ 10.1007/3-540-44618-4_9

32. Kupferman, O., Piterman, N., Vardi, M.Y.: An automata-theoretic approach to infinite-state systems. In: Manna, Z., Peled, D.A. (eds.) Time for Verification. LNCS, vol. 6200, pp. 202-259. Springer, Heidelberg (2010). https://doi.org/10. 1007/978-3-642-13754-9_11

33. Kupferman, O., Vardi, M.Y.: An automata-theoretic approach to reasoning about infinite-state systems. In: Emerson, E.A., Sistla, A.P. (eds.) CAV 2000. LNCS, vol. 1855, pp. 36-52. Springer, Heidelberg (2000). https://doi.org/10.1007/10722167_7

34. Löding, C., Madhusudan, P., Neider, D.: Abstract learning frameworks for synthesis. In: Chechik, M., Raskin, J.F. (eds.) LTACAS 2016. LNCS, vol. 9636, pp. 167-185. Springer, Heidelberg (2016). https://doi.org/10.1007/978-3-662-496749_10

35. Madhusudan, P.: Synthesizing reactive programs. In: CSL. LIPIcs, vol. 12, pp. 428-442. Schloss Dagstuhl - Leibniz-Zentrum fuer Informatik (2011). https://doi. org/10.4230/LIPIcs.CSL.2011.428

36. Madhusudan, P., Mathur, U., Saha, S., Viswanathan, M.: A decidable fragment of second order logic with applications to synthesis. In: Ghica, D., Jung, A. (eds.) 27th EACSL Annual Conference on Computer Science Logic (CSL 2018). Leibniz International Proceedings in Informatics (LIPIcs), vol. 119, pp. 31:1-31:19. Schloss Dagstuhl-Leibniz-Zentrum fuer Informatik, Dagstuhl (2018). https://doi.org/10. 4230/LIPIcs.CSL.2018.31

37. Madhusudan, P., Parlato, G.: The tree width of auxiliary storage. In: Proceedings of the 38th Annual ACM SIGPLAN-SIGACT Symposium on Principles of Programming Languages, POPL 2011, pp. 283-294. ACM, New York (2011). https:// doi.org/10.1145/1926385.1926419

38. Madhusudan, P., Thiagarajan, P.S.: Distributed controller synthesis for local specifications. In: Orejas, F., Spirakis, P.G., van Leeuwen, J. (eds.) ICALP 2001. LNCS, vol. 2076, pp. 396-407. Springer, Heidelberg (2001). https://doi.org/10.1007/3540-48224-5_33

39. Mathur, U., Madhusudan, P., Viswanathan, M.: Decidable verification of uninterpreted programs. Proc. ACM Program. Lang. 3(POPL), 46:1-46:29 (2019). https://doi.org/10.1145/3290359

40. Mathur, U., Madhusudan, P., Viswanathan, M.: What's decidable about program verification modulo axioms? In: Biere, A., Parker, D. (eds.) TACAS 2020. LNCS, vol. 12079, pp. 158-177. Springer, Cham (2020). https://doi.org/10.1007/978-3030-45237-7_10

41. Mathur, U., Murali, A., Krogmeier, P., Madhusudan, P., Viswanathan, M.: Deciding memory safety for single-pass heap-manipulating programs. Proc. ACM Program. Lang. 4(POPL), 1-29 (2019). https://doi.org/10.1145/3371103

42. Müller-Olm, M., Rüthing, O., Seidl, H.: Checking herbrand equalities and beyond. In: Cousot, R. (ed.) VMCAI 2005. LNCS, vol. 3385, pp. 79-96. Springer, Heidelberg (2005). https://doi.org/10.1007/978-3-540-30579-8_6

43. Muscholl, A., Walukiewicz, I.: Distributed synthesis for acyclic architectures. In: FSTTCS. LIPIcs, vol. 29, pp. 639-651. Schloss Dagstuhl - Leibniz-Zentrum fuer Informatik (2014). https://doi.org/10.4230/LIPIcs.FSTTCS.2014.639

44. Pnueli, A., Rosner, R.: On the synthesis of a reactive module. In: POPL, pp. 179190. ACM Press (1989). https://doi.org/10.1145/75277.75293 
45. Pnueli, A., Rosner, R.: Distributed reactive systems are hard to synthesize. In: FOCS, pp. 746-757. IEEE Computer Society (1990). https://doi.org/10.1109/ FSCS.1990.89597

46. Post, E.L.: A variant of a recursively unsolvable problem. Bull. Amer. Math. Soc. 52(4), 264-268 (1946). https://doi.org/10.1090/S0002-9904-1946-08555-9

47. Qiu, X., Solar-Lezama, A.: Natural synthesis of provably-correct data-structure manipulations. PACMPL 1(OOPSLA), 65:1-65:28 (2017). https://doi.org/10. $1145 / 3133889$

48. Rabin, M.O.: Automata on Infinite Objects and Church's Problem. American Mathematical Society, Boston (1972)

49. Singh, R., Gulwani, S., Solar-Lezama, A.: Automated feedback generation for introductory programming assignments. SIGPLAN Not. 48(6), 15-26 (2013). https:// doi.org/10.1145/2499370.2462195

50. Solar-Lezama, A.: Program sketching. Int. J. Softw. Tools Technol. Transf. 15(5), 475-495 (2013). https://doi.org/10.1007/s10009-012-0249-7

51. Solar-Lezama, A., Tancau, L., Bodík, R., Seshia, S.A., Saraswat, V.A.: Combinatorial sketching for finite programs. In: ASPLOS, pp. 404-415. ACM (2006). https:// doi.org/10.1145/1168857.1168907

52. SyGuS: Syntax guided synthesis. https://sygus.org/

53. Vardi, M.Y.: Reasoning about the past with two-way automata. In: Larsen, K.G., Skyum, S., Winskel, G. (eds.) ICALP 1998. LNCS, vol. 1443, pp. 628-641. Springer, Heidelberg (1998). https://doi.org/10.1007/BFb0055090

54. Wang, X., Dillig, I., Singh, R.: Program synthesis using abstraction refinement. Proc. ACM Program. Lang. 2(POPL), 63:1-63:30 (2017). https://doi.org/10.1145/ 3158151

55. Wang, X., Gulwani, S., Singh, R.: FIDEX: filtering spreadsheet data using examples. In: Proceedings of the 2016 ACM SIGPLAN International Conference on Object-Oriented Programming, Systems, Languages, and Applications, OOPSLA 2016, pp. 195-213. ACM, New York (2016). https://doi.org/10.1145/2983990. 2984030

56. Wang, Y., Wang, X., Dillig, I.: Relational program synthesis. Proc. ACM Program. Lang. 2(OOPSLA), 155:1-155:27 (2018). https://doi.org/10.1145/3276525

Open Access This chapter is licensed under the terms of the Creative Commons Attribution 4.0 International License (http://creativecommons.org/licenses/by/4.0/), which permits use, sharing, adaptation, distribution and reproduction in any medium or format, as long as you give appropriate credit to the original author(s) and the source, provide a link to the Creative Commons license and indicate if changes were made.

The images or other third party material in this chapter are included in the chapter's Creative Commons license, unless indicated otherwise in a credit line to the material. If material is not included in the chapter's Creative Commons license and your intended use is not permitted by statutory regulation or exceeds the permitted use, you will need to obtain permission directly from the copyright holder. 